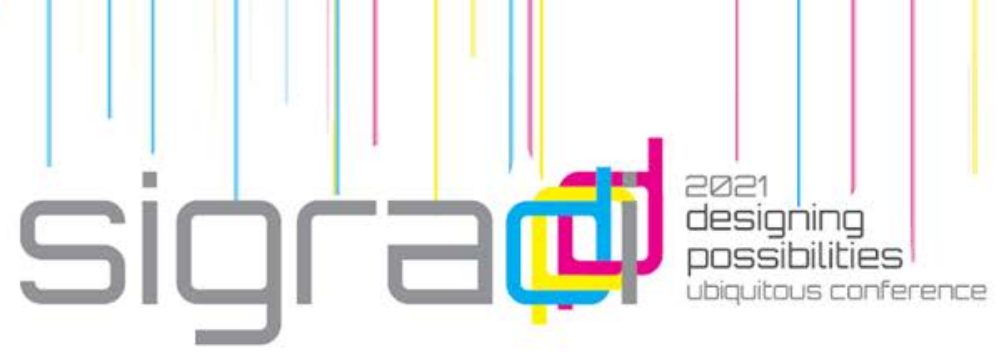

\title{
Quantification of Effective Temporal Exposure to Daylight Illuminance Levels in Healthcare Settings
}

\author{
Marcelo Bernal, Ph.D. ${ }^{1}$, Gonzalo Vegas ${ }^{2}$, Marvina Williams $^{1}$, Katie Andersen ${ }^{1}$ \\ ${ }^{1}$ Perkins\&Will, United States \\ Marcelo.Bernal@perkinswill.com \\ Marvina.Williams@perkinswill.com \\ Katie.Andersen@perkinswill.com \\ ${ }^{2}$ Georgia Institute of Technology, United States \\ gvegas3@gatech.edu
}

\begin{abstract}
The purpose of this study is the quantification of the exposure of occupants to daylight illuminance levels. The case study is the typical floor of a patient tower occupied by nurses in twelve hours shifts, from 7 am to $7 \mathrm{pm}$. Significant evidence exists regarding the positive impact of access to daylight on staff outcomes in healthcare facilities in terms of reduction of stress, absenteeism, medication errors, and burn outs. However, the standard daylight simulation methods evaluate the building and do not capture the dynamic nature of people's behavior while moving through the space. The proposed approach combines agent-based simulation and daylight performance analysis to compute the occupants' exposure to daylight levels throughout the year. The results show the discrepancies between building-centric and human-centric types of analysis and the contribution of dynamic simulation methods to design occupancy schedules to warranty equitable access to daylight to building occupants.
\end{abstract}

Keywords: Agent-based simulation, Performance-based design, Spatial occupancy, Human-centric analysis

\section{Introduction}

A multitude of research has been done on analyzing the building performance achieving high levels of resolution and reliable results. Unlike energy analysis, which is based on thermal zones, most of the environmental analyses are gridbased. These analyses define an analysis plane, at a certain height, a grid of points, evaluate the intensity levels for a given metric per sensor point, and visualize results through heatmaps. Even though grid-based analyses effectively characterize performance, they are building-centric and do not necessarily capture occupants' exposure to the environmental conditions they 
measure. Occupants behave like moving sensors, constantly changing positions and orientations through space and time. Consequently, occupant's comfort cannot be accurately calculated solely relying on these grid-based analyses. The characterization of the spatiotemporal occupancy of the space remains an active area of research (Gomez Zamora, 2017).

On the other hand, several efforts have been made to simulate realistic user occupation of buildings through a combination of Discrete Event and Agentbased systems (Heath, et al. 2011). This approach represents variations in position and orientation of each occupant through time, which has been tested in usability and accuracy to represent human behavior (Luo, et al. 2017). These simulations rely on agents representing different occupant roles and interactions and schedules embedding the expected sequence of actions they are supposed to perform along with a specific time frame such as daily shifts, weeks, or even the entire year. These simulation models can be based either on predefined schedules or data based on real measurements of people's behavior. They provide valuable information to predict the actual use of the space. Several implementations have been made with increasing levels of complexity across different fields. Initially, in highly abstracted proofs of concepts to optimize space planning (Shen, et al, 2010). They were later developed together with pedestrian simulation as a tool to improve designerclient communication (Shen, et al. 2013) and evaluate occupancy performance indicators such as path lengths and density (Schaumann, et al, 2019). And finally, allowing designers to assess behavioral consequences of design decisions (Schaumann, et al. 2020).

We can find examples of Pre-Occupancy Evaluations (PrOE) in the field of energy analysis (Hong, et al. 2015), mainly to evaluate more realistic building energy consumption (Chen, et al. 2017), or in small-scale user-space interactions (Shin, et al., 2016). Gomez-Zamora et al. (2019) also demonstrated the radical influence of the caregivers' circulation paths and schedules on the accumulated time of unplanned surveillance patients receive, affecting patient outcomes. However, there is still ground to cover to dynamically evaluate the occupant's comfort levels in other areas such as Daylight, Glare, Thermal Comfort, or Views of the Outdoors.

To measure occupants' exposure to environmental conditions, specifically Daylight Illuminance, this proposed approach merges both building performance analysis and occupant's behavior simulations in an integrated process. We tried our approach in a healthcare facility for Critical and MedicalSurgical Care Units to quantify the exposure to the daylight of healthcare providers during working hours. Unlike inpatients that spend a fair amount of time in bed in a fixed location in these units, healthcare providers are constantly moving from one location to another during the long twelve-hour seven-toseven shifts.

The relevance of accurately tracking the actual exposure of caregivers to daylight is due to the significant influence on staff outcomes and, subsequently, on the patients' direct benefits. For example, Alimogli \& Donmez (2005) 
surveyed the positive effect of exposure to sunlight for more than three hours on reducing nurses' work-related levels of stress and increasing job satisfaction. Ulrich et al. (2008) published an extensive review of evidencebased design in healthcare literature that shows the correlations, not necessarily causality, between environmental factors and staff and patient outcomes. They classified the results of several research efforts and reported the impact of illuminance levels on different areas. The results show medication errors rate reductions from $3.8 \%$ to $2.6 \%$ while increasing daylight intensity from 450 to 1500 lux (Buchanan et al.,1991). In the same report, Lepparmaki et al. (2003) show the impact of repeated brief exposure (4×20 minutes) to bright light over 5000 lux on self-reported well-being of night shift nurses, and Partonen \& Lonnqvist (1998) found that treatment with bight over light 2500 lux has a positive impact on distress associated with night shifts and even mood in healthy people. In terms of visual tasks, Figueiro et al. (2006) measured the performance benefits at the working plane starting at $600 \mathrm{lux}$, and Zadeh et al. (2014) documented the benefits of the presence of sunlight on physiological responses, improved communication, and even on the overall mood of the healthcare providers while comparing the performance of nurses assigned to windowed versus windowless locations.

More recently, assuming the positive impact of access to daylight on the staff outcomes. Hadi and Pewzer (2018) quantified the duration of the exposure to daylight by comparing the annual average illuminance levels per space and the amount of time the caregivers stayed in each room. For this purpose, they implemented a Discrete Event Simulation model based on schedules and data collected on-site to simulate staff behavior in a module of twelve exam rooms. The results showed the staff experiencing far less than three hours of daylight exposure, compromising their overall performance. They also pointed out the need to increase the accuracy of the analysis at higher levels of granularity to create a more realistic evaluation of such a critical aspect. In this regard, the purpose of this study is to measure the caregiver's exposure to daylight at the resolution of two-by-two square feet every hour throughout the entire year. The aim is to capture the slightest variation in daylight intensity in space and time.

The proposed methodology also integrates both behavioral and environmental simulations in a single process. This approach executes two types of simulations: a performance analysis to characterize the space's environmental conditions and a multi-agent narrative model to represent the space occupancy. While we simulate the occupant's movement across space, we link the position and orientation to the environmental information to calculate the accumulated exposure and plot the fluctuations along the year at the grid level. The results show significant variations in daylight exposure of caregivers assigned to the same floor, performing a similar task, but in different building orientations. 


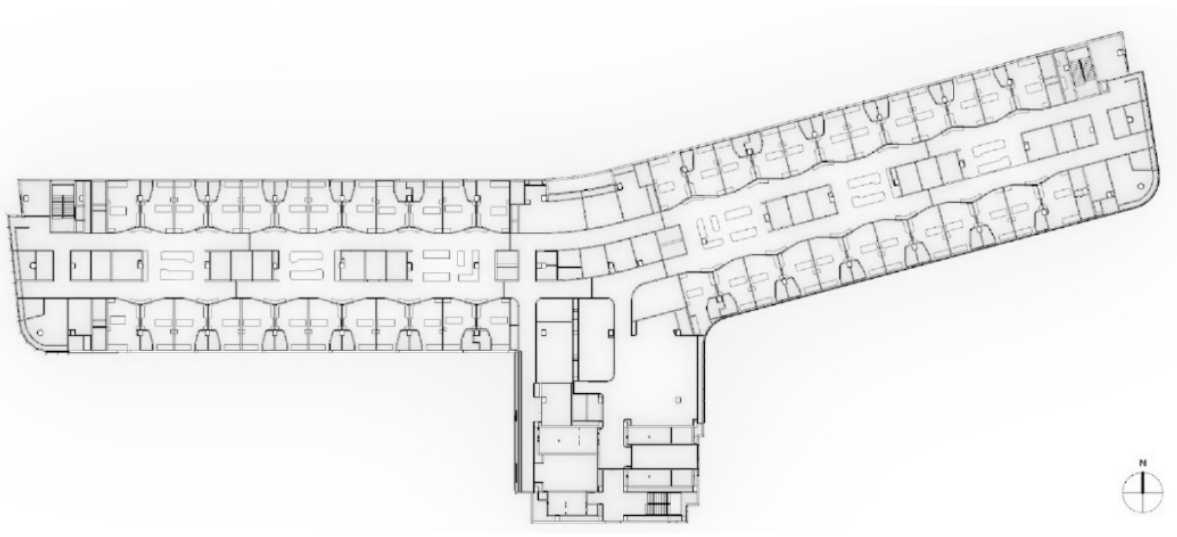

Figure 1. Critical Care Units to the left and Medical-Surgical Care Units to the right.

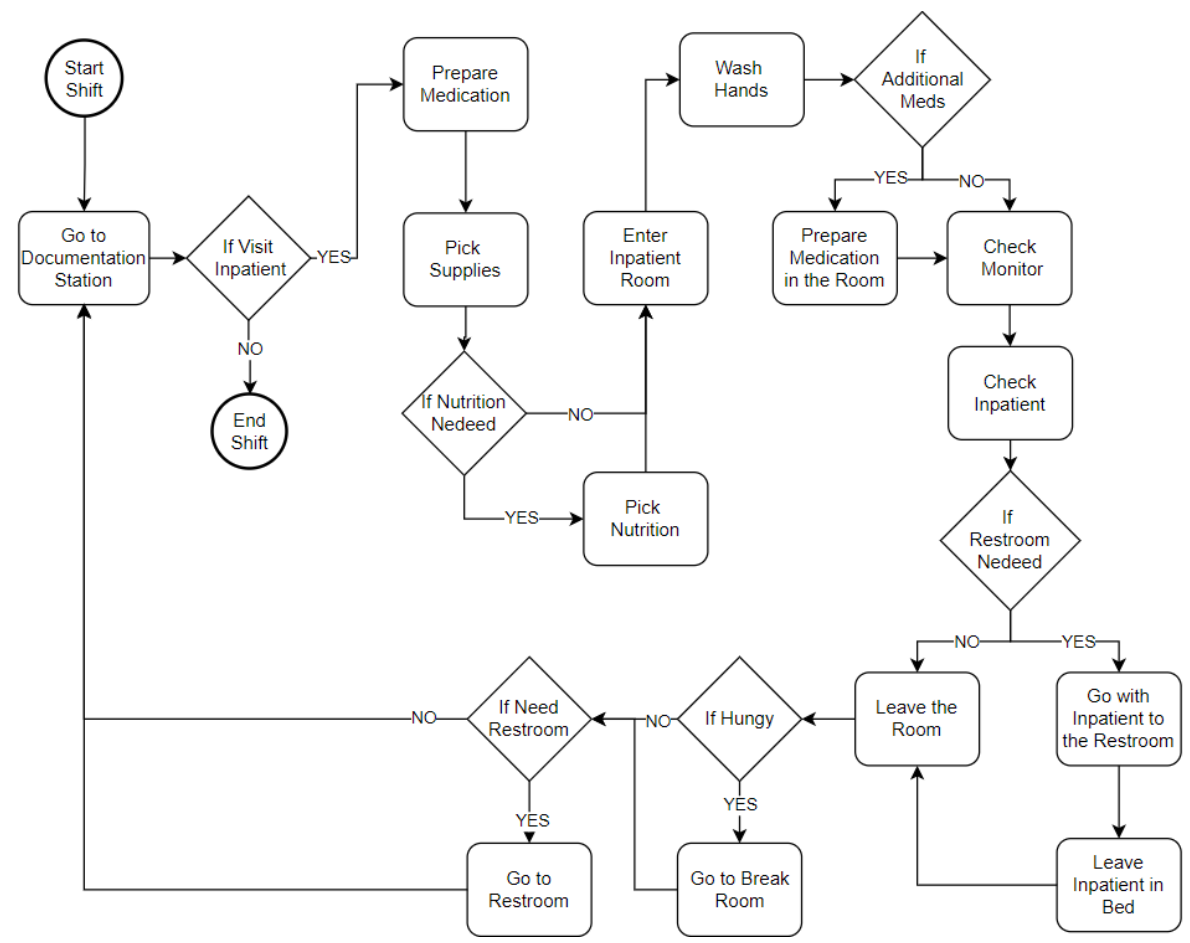

Figure 2. Activity diagram representing the sequence of actions and decisions nurses make every time they visit inpatients during twelve hours shifts. 


\subsection{Daylight Exposure}

To calculate nurses' exposure to daylight illuminance levels larger than 300 , 600, and 1500 lux, firstly, we use Climate Studio (Solemma, 2020) to run the annual daylight simulations with a grid of sensor points two-by-two feet at the working plane (Fig.5). The data contains values for each sensor every hour of the entire year (Fig.6). A second post-process calculates the intersection in space and time with the agents representing nurses to compute their hourly exposure. To do so, we record the agents' positions in the grid each second during the twelve-hour shift together with their corresponding timestamps, resulting in 43,200 total locations for each agent every day. Later, we identify the closest hour and grid point to each agent position to obtain the illuminance value. This process is executed for the 365 days of the year. To plot the results, we average the exposure of all the nurses per zone.

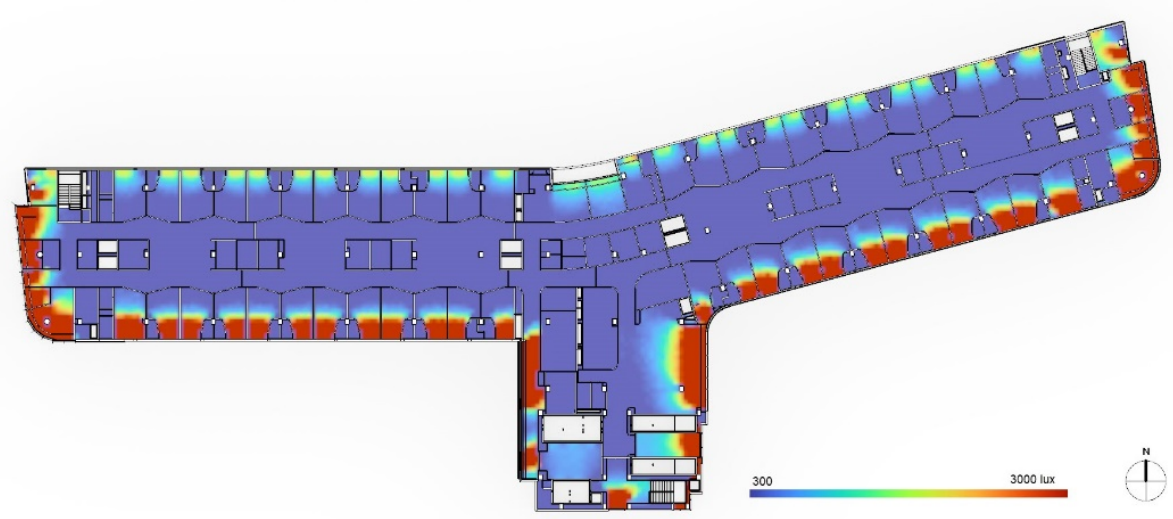

Figure 5. Annual illuminance average. Below 300lux blue, above 3000lux red.

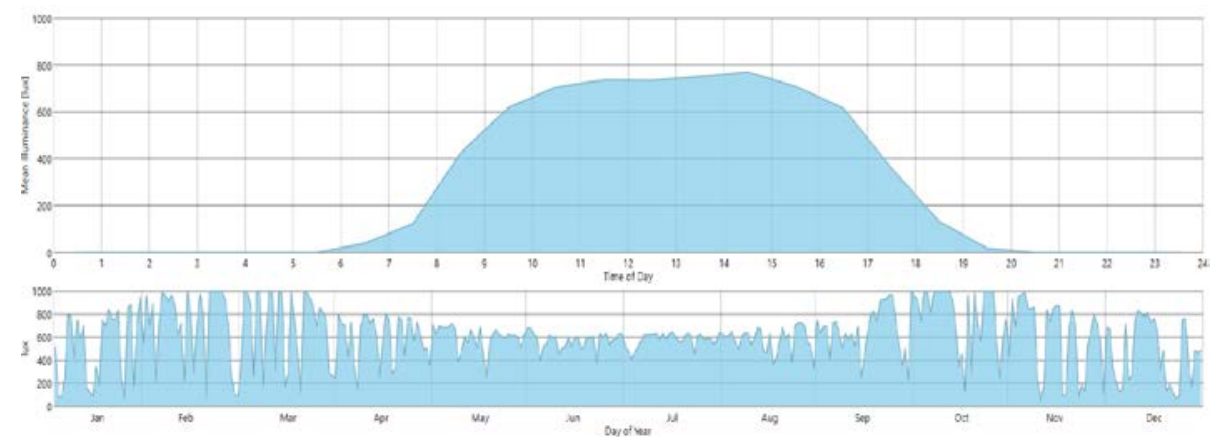

Figure 6. Overall daily mean illuminance above, mean illuminance for 365 days below. 

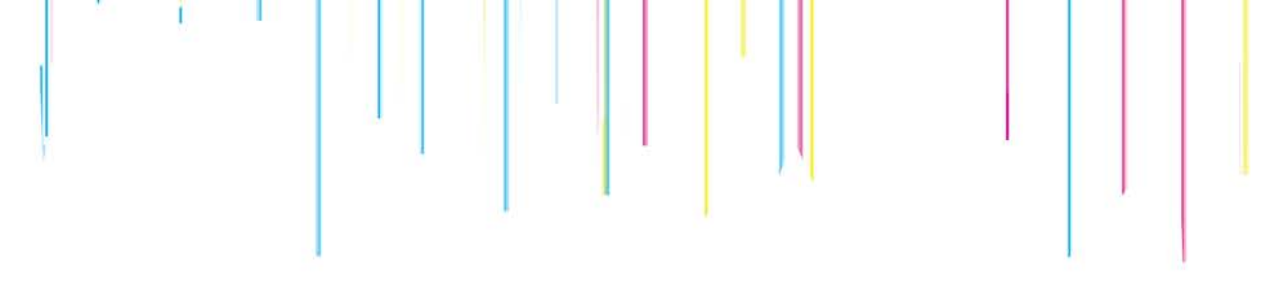

\section{Results}

We implemented narratives for nurses that constantly navigate through the space. We record each movement and link their position and direction with the results of the adjacent sensor points. Once the simulation is complete, we obtain each occupant's accumulated temporal exposure to different thresholds of 300,600, and 1500 lux during shifts (Fig.7) throughout the entire year.

Stress reduction and job satisfaction occurs when the accumulated daylight exposure is more than three hours a day. The minimum threshold we can associate with the presence of daylight is 300lux. The 300lux threshold (Fig.8) shows that nurses in the Medical-Surgical Care East wing are exposed most days above the three-hour threshold in both orientations. The illuminance levels in the Medical-Surgical Care wing are higher than in the Critical Care due to the slight rotation of the floor plan on the Eastside. Nevertheless, the West wing seems close to the desired threshold for the two groups in that area.

The 600lux threshold (Fig.9) that correlates with visual tasks shows Critical Care South around 1.5 hours per day and Medical-Surgical Care Southeast 2.4 hours per day most of the year, which is remarkable. However, Critical Care North and Medical-Surgical Care Northwest show an uneven distribution concentrating the higher exposure in the center half of the year reaching 0.6 and 1.2 hours per day, respectively, exposing the need for artificial lighting to support visual tasks the rest of the year.

The 1500lux threshold (Fig.10), which correlates with reducing medication errors, shows lower durations in the four groups. While the Critical Care North and Medical-Surgical Care Northwest groups achieve no time within the threshold, the Critical Care South and the Medical-Surgical Care Southeast achieve almost half an hour in the beginning and end of the year.

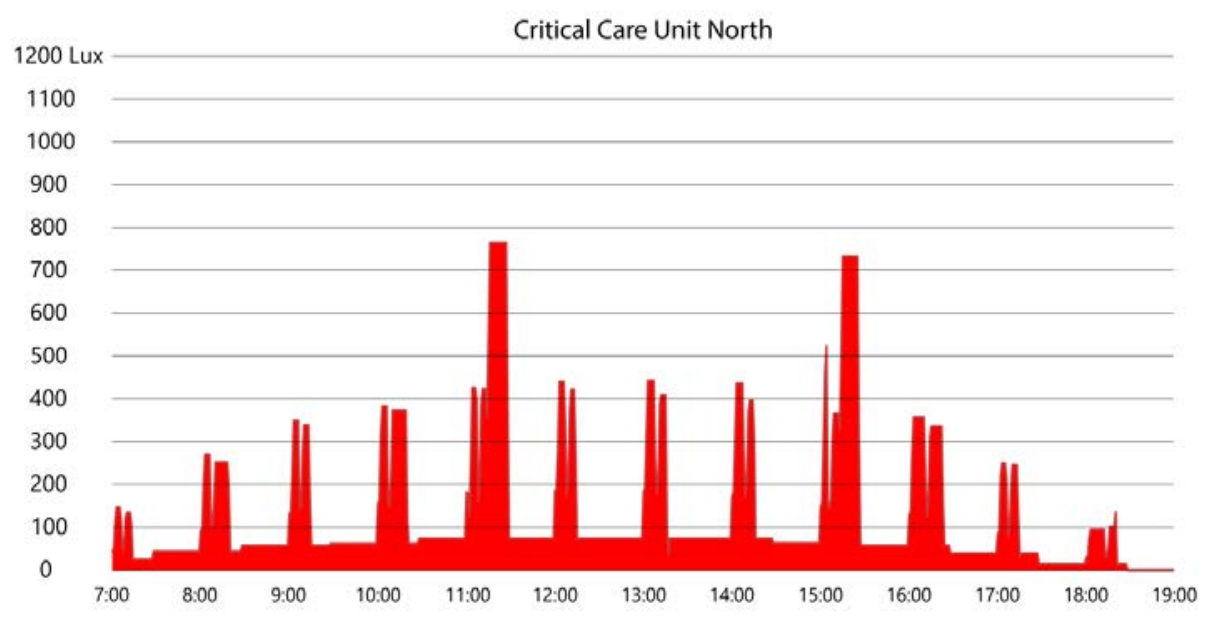

Figure 7. Exposure of Nurse $\mathrm{N}^{\circ} 04$ during the $7 \mathrm{am}$ to $7 \mathrm{pm}$ shift on March $21^{\text {th }}$. 
Consistently, across the three evaluated thresholds, the four groups show radically different levels of exposure while executing similar tasks on the same floor. The discrepancies across nurse groups highlight the importance of the design of the occupancy schedule from an equity perspective. Even though Fig. 7 is a sample of one shift of one nurse on a specific day, it is a clear example of nurses exposed to higher illuminance levels while are in the inpatient room. This exposure in the inpatient rooms is consistent with Fig.6, which shows the corridors and other spaces at 300lux and below, except for the break room.

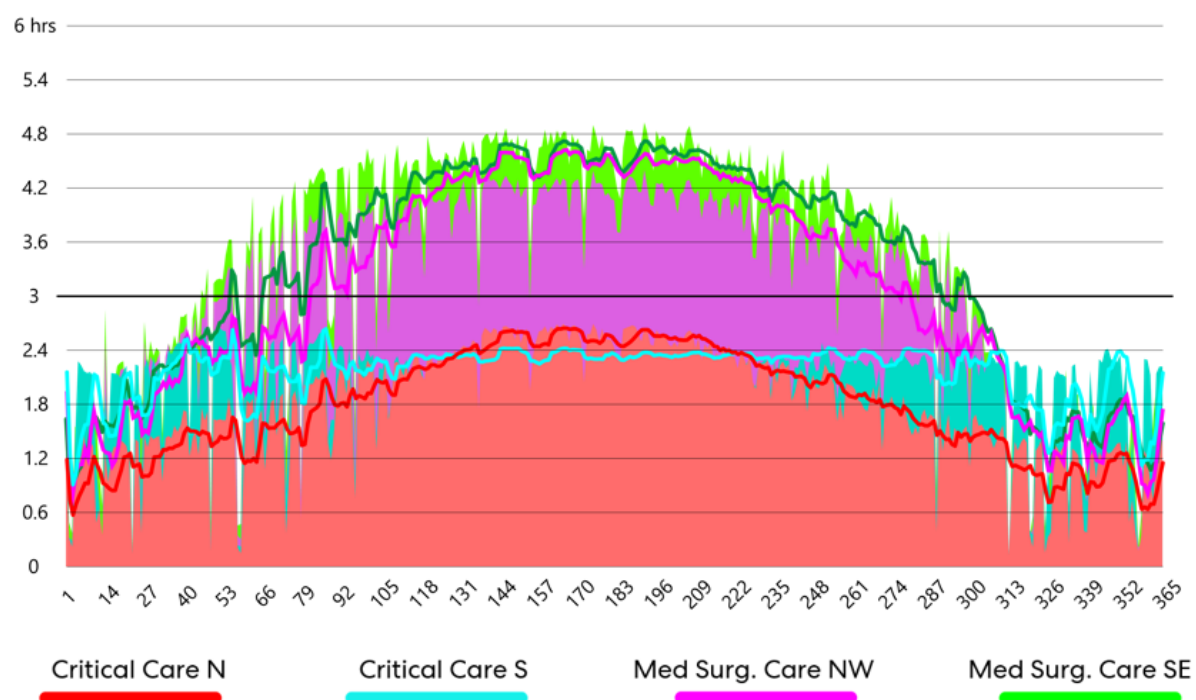

Figure 8. Seven days average time over 300lux per zone across 365 days.

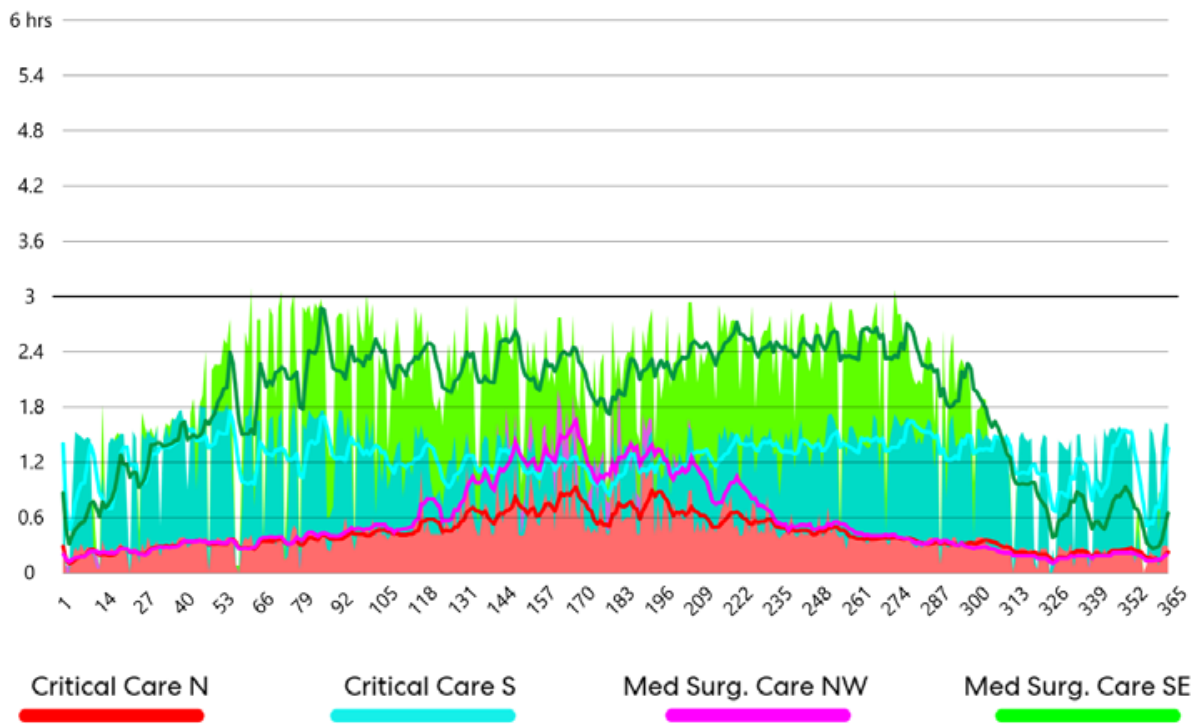

Figure 9. Seven days average time over 600lux per zone across 365 days 


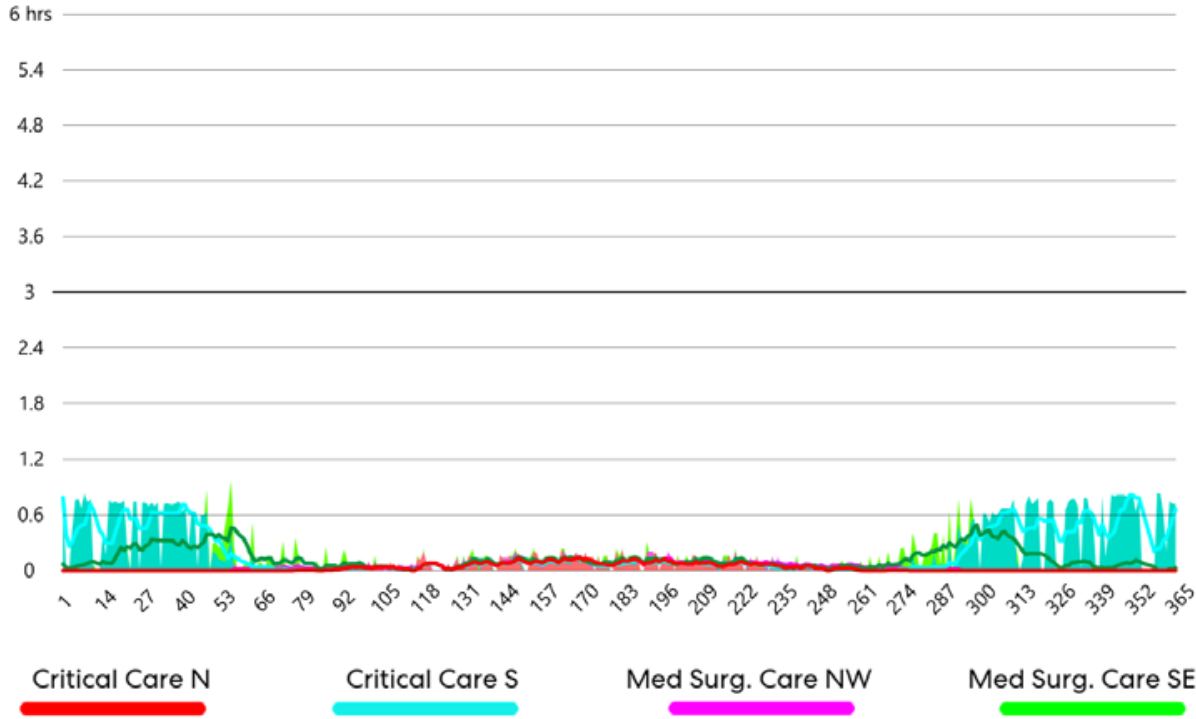

Figure 10. Seven days average time over 1500lux per zone across 365 days

\section{Discussion}

This approach proposes the migration from a building-centric to a humancentric type of analysis by quantifying and demonstrating the potential differences to exposure to environmental conditions among different occupants of the same facility. Even though the general characterization of the building performance provides a reliable context to make assumptions regarding the quality of the space, discrepancies in terms of schedules can situate similar occupants sharing similar tasks in different conditions. Despite the dependency on the schedules' accuracy or the limitations to represent the unplanned behaviors, the proposed method provides valuable feedback to anticipate the actual occupancy of the space. The occupancy, or the schedule driving the occupancy, is a design itself. While the annual daylight performance of the building remains constant, the design of the occupancy schedules in such a strong program can be adapted to distribute the access to daylight and other environmental factors equitably.

Considering the amount of data per agent by location, intensity, hour, shifts across the entire year, and the variety of questions regarding the combination of illuminance levels, time of the day, accumulated exposure, and impact of specific tasks, a data visualization interface seems to be the logical next step in this research to support the interpretation of the results. 


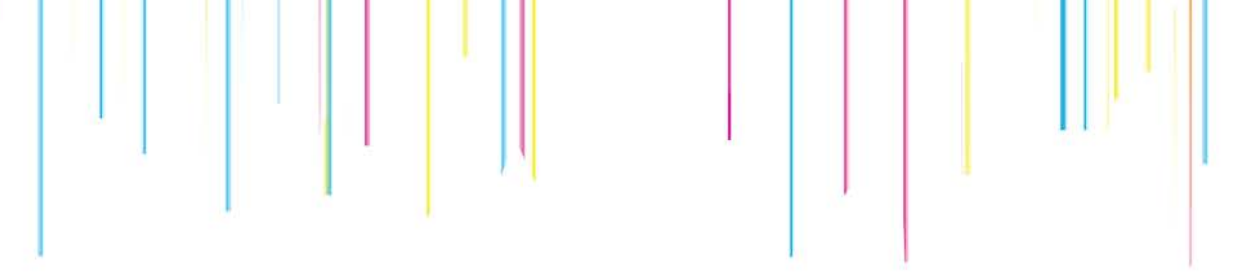

Acknowledgements. We want to thank the Perkins\&Will Research Board and the Healthcare Practice Leaders for their support and advice in this research.

\section{References}

Alimoglu, M. K., \& Donmez, L. (2005). Daylight exposure and the other predictors of burnout among nurses in a university hospital. International Journal of Nursing Studies, 42(5), 549-555. doi:http://dx.doi.org/10.1016/j.ijnurstu.2004.09.001

Buchanan, T. L., Barker, K. N., Gibson, J. T., Jiang, B. C., \& Pearson, R. E. (1991). Illumination and errors in dispensing. American Journal of Hospital Pharmacy, 48(10), 2137-2145. https://doi.org/10.1093/ajhp/48.10.2137

Chen, Y., Liang, X., Hong, T., \& Luo, X. (2017). Simulation and visualization of energyrelated occupant behavior in office buildings: Building Simulation.

Daniel, K., Nash, A., \& Koenig, S. (2010). Theta*: Any-Angle Path Planning on Grids. Journal of Artificial Intelligence Research, 39, 533-579.

Gomez Zamora, P. A. (2017). Spatiotemporal occupancy in building settings [Ph.D. Thesis, Georgia Institute of Technology]. Atlanta.

Gomez-Zamora, P., Bafna, S., Zimring, C., Do, E., \& Romero, M. (2019). Spatiotemporal Occupancy for Building Analytics. Proceedings of the 37th eCAADe and 23rd SIGraDi Conference, Porto, Portugal.

Hadi, K., \& Pewzer, O. (2018). Predicting Duration of Daylight Exposure for Caregivers Using Discrete Event Simulation and Climate-Based Daylight Modeling. Paper presented at the Symposium on Simulation for Architecture and Urban Design, Delft, Netherlands.

Heath, S. K., Brailsford, S. C., Buss, A., \& Macal, C. M. (2011). Cross-paradigm simulation modeling: challenges and successes. Proceedings of the 2011 winter simulation conference (WSC)

Hong, T., Sun, H., Chen, Y., Taylor-Lange, S. C., \& Yan, D. (2016). An occupant behavior modeling tool for co-simulation. Energy and Buildings, 117, 272-281.

Leppämäki, S., Partonen, T., Piiroinen, P., Haukka, J., \& Lönnqvist, J. (2003). Timed bright-light exposure and complaints related to shift work among women. Scandinavian journal of work, environment \& health, 22-26.

Luo, X., Lam, K. P., Chen, Y., \& Hong, T. (2017). Performance evaluation of an agentbased occupancy simulation model. Building and Environment, 115, 42-53.

Naderpour, A., Johnson, B., \& Anderson, A. (2019). A2B: A Toolkit for Computing Circulation Metrics in Buildings. IBPSA.

Partonen, T., \& Lönnqvist, J. (1998). Seasonal affective disorder. CNS drugs, 9(3), 203212.

Schaumann, D., Breslav, S., Goldstein, R., Khan, A., \& Kalay, Y. (2017). Simulating the Behavior of Building Occupants using Multi-agent Narratives. Building Simulation, San Francisco, CA, USA, 
Schaumann, D., \& Kapadia, M. (2019). Modeling Social and Spatial Behavior in Built Environments: Current Methods and Future Directions. In P. Davis, A. O'Mahony, \& J. Pfautz (Eds.), Social-Behavioral Modeling for Complex Systems (pp. 673-695). Stevens Institute Series on Complex Systems and Enterprises.

Shen, W., Zhang, X., Qiping, G., \& Terrence, F. (2013). The User Pre-Occupancy Evaluation Method in designer-client communication in early design stage: A case study. Automation in Construction, 32, 112-124.

Shin, S., Jeong, S., Lee, J., Hong, S. W., \& Jung, S. (2017). Pre-Occupancy Evaluation based on user behavior prediction in 3D virtual simulation. Automation in Construction, 74, 55-65.

Solemma. (2020). ClimateStudio. Retrieved April from https://www.solemma.com/ClimateStudio.html

Shen, W., \& Shen, Q. (2010). User Activity Simulation and Evaluation Model for the Improvement of Space Planning in Office Buildings. W101-Special Track 18th CIB World Building Congress May 2010 Salford, United Kingdom,

Ulrich, R. S., Zimring, C., Zhu, X., DuBose, J., Seo, H.-B., Choi, Y.-S., Quan, X., \& Joseph, A. (2008). A review of the research literature on evidence-based healthcare design. HERD: Health Environments Research \& Design Journal, 1(3), 61-125.

U.S. Green Building Council (2021). LEED v4. Retrieved from https://www.usgbc.org/leed/v4

Wang, P. (2016). Understanding social-force model in psychological principles of collective behavior. arXiv preprint arXiv:1605.05146.

Zadeh, R. S., Shepley, M. M., Williams, G., \& Chung, S. S. E. (2014). The impact of windows and daylight on acute-care nurses' physiological, psychological, and behavioral health. HERD: Health Environments Research \& Design Journal, 7(4), 35-61. 